\title{
Augmenting Social Media Research with Q Methodology: Some Guiding Principles
}

\author{
Charmaine du Plessis \\ University of South Africa, Pretoria, South Africa \\ dplestc@unisa.ac.za \\ DOI: 10.34190/JBRM.17.3.005
}

\begin{abstract}
This paper proposes that social media studies could be complemented with Q methodology when a topic that plays out in social media is complex, controversial or sensitive to allow for deep-seated, integrated online and off-line perspectives. Although the Fourth Industrial Revolution brought researchers more opportunities and advantages to study topics that were previously inaccessible, using technologies for research does not come without challenges. This is especially the case with social media studies comprising large datasets and where it is not always possible to identify fake profiles, bots, spam or manipulated information without having access to advanced data analysis software. Another point is that views expressed in social media do not always represent offline perspectives. However, while $Q$ methodology has, over the years, adapted its techniques to accommodate new technologies, more can be done to embrace a web 2.0 environment. Why and how social media studies could be augmented with $Q$ methodology to reveal individuals' perspectives and attitudes about topics will be examined and potential difficulties will be highlighted. Not yet a mainstream method, $Q$ methodology combines the strengths of two robust qualitative and quantitative methods sequentially to reveal and isolate the subjective perspectives of groups of participants. This methodology could, therefore, be useful when a social media study puts forward novel ideas and findings that should be supported by offline views. In this regard, the paper provides some guidelines by referring to the five phases of a $Q$ study and describing how a social media study could not only benefit from but also apply $Q$ methodology to augment results. Supplementing social media research with $Q$ methodology could be empowering and provide opportunities for further research and debate.
\end{abstract}

Keywords: mixed method, social media research, Q factor analysis, Q methodology, Q study

\section{Introduction}

The Fourth Industrial Revolution (4IR) has brought more opportunities for research than ever before since digital technologies create unlimited and speedy access to information and content. Although 4IR is about emerging technologies and how they blend into the physical world, it is also about the size, speed and scope of change and access to knowledge (Schwab, 2016). 4IR changed not only how researchers work, but also how they express themselves and obtain data for analysis with more opportunities for knowledge sharing since technologies connect people and ideas globally (Shalin, 2015). Researchers nowadays have considerable advantages because of the speed of and greater access to technology and connectivity, but these also pose challenges (Sappleton, 2013).

Some of the persisting challenges are methodological issues facing academic researchers who conduct social media research. Especially the way in which researchers gather observational social media data is questioned since datasets often do not account for all variables in a study (Stephen, 2014; Poynter, 2018). Social media data also do not necessarily reflect the larger online and offline population because of manipulation in the form of fake accounts, spam posts, bots, activists or haters creating a hashtag trend through coordinated efforts. Poynter (2011) refers to "complicit participants" as it is not always possible for a researcher collecting a vast amount of social media data to detect manipulation (Tufekci, 2014) unless advanced data analysis software, which requires some technical expertise, is used (Zheng et al., 2015). Research by Beninger et al. (2014) indicates that social media users also question the validity and representativeness of social media research because social media does not reflect their real world. The anonymity of some social media networks, for example, often encourages exaggerated views and behaviour that differ from those held and displayed offline. Also, comments could be made at the spur of the moment that do not reflect the general view.

Although this might be true, it would be wrong to claim that all social media research lacks validity. There is evidence of meticulous social media research with findings that contribute to the body of knowledge. Using social media data are essential for 4IR research since they constitute an important part of a society's functioning and are tied to business and politics as well as everyday life and relationships (Burgess, Marwick and Poell, 2017). Important to realise is that some social media data are more complex, controversial or 
sensitive than others and, as a result, could attract more spam and complicate a study's results (Inuwa-Dutse, Liptrott and Korkontzelos, 2018).

This paper puts forward the idea that, in cases where observational social media datasets could be unsound, researchers conducting social media research could enhance their studies by adding $\mathrm{Q}$ methodology as an additional phase. It is well known that some topics attract more attention than others in social media, which could taint a social media dataset with undetectable fake users and spam (Tufekci, 2014). The advantages of using $Q$ methodology are twofold. Firstly, it can be argued that while social media conversations reflect more honest views than, for example, during interviews (see Beninger et al., 2014), Q methodology provides participants with the opportunity to share deep-rooted and thoughtful views on difficult topics without fear of embarrassment or identification. Their views could benefit the results of social media research as online and offline views become integrated. The methodology also provides the researcher with a robust tool to validate novel ideas and findings based on observational social media data that could be comprised of questionable and undetectable social media profiles, posts and images.

Combining $\mathrm{Q}$ methodology with more traditional methodologies is nothing new. It is often done for surveys and interviews (see Stergiou and Airey, 2011), and could also be beneficial for social media studies. Whereas numerous Q studies about social media exist (see Stephen, 2014; Orchard et al., 2015; Thomson and Greenwood, 2017), no research using large and raw social media datasets has been conducted. While there is a keen and increased interest in what $Q$ methodology has to offer, especially novel researchers struggle with the practicalities of a $Q$ study (Ramlo, 2016). Hence it is acknowledged that combining social media datasets with $\mathrm{Q}$ methodology could be challenging since the methodology has not fully adapted to a social media environment. $Q$ methodology could nevertheless offer a valuable addition to social media research since its robust techniques will use the strengths of both the qualitative and quantitative approaches to study the subjective, integrated online and offline perspectives of participants about important topics that play out in social media. It will be possible to isolate and validate the dominant perceptions that are evident in a large social media dataset (see Brown, 1980).

This article provides some guidelines on how a social media study could not only benefit from but also apply $Q$ methodology to enhance results. As an exploratory, inductive and deductive methodology, $\mathrm{Q}$ methodology strengthens subjective viewpoints with the statistical operation of $Q$ factor analysis (Ramlo, 2016).

The paper is structured as follows. First, Q methodology is explained and some of the methodology's advantages and disadvantages are highlighted. This is followed by an explanation of how social media researchers could use this methodology to enhance the results of social media studies, and a discussion of some difficulties. The paper ends with some conclusions and recommendations.

\section{What is $Q$ methodology?}

Underpinned by a post-positivistic research paradigm (Killam, Timmermans and Raymond, 2013; Ramlo and Newman, 2011), Q methodology (also referred to as a Q study) is not a new methodology. In fact, it dates back to 1935. Initially applied in the field of psychology, this methodology has been adopted across the globe in other fields such as nursing education (Henzel, 2016), the health sciences (Richardson, 2016), education (Grover, 2015), social work (Ellingsen, Størksen and Stephens, 2010), branding (Le Roux and Du Plessis, 2014), business studies (Angelopulo, 2009) and identity and gender studies (Sung-Hee, Euna and Myung-Ae, 2014), to name a few.

Although not as well-known as other more conventional research methods, it is evident that Q methodology at present is globally applied in academic studies across various disciplines with an active and helpful online community, an academic journal and annual conferences (Ramlo, 2016). Despite it being criticised as "overly ambitious" (Kampen and Tamás, 2014) and "qualiquantological" (Parker and Alford, 2010), the body of knowledge and application of $Q$ methodology are steadily increasing as the pragmatism of this methodology is more widely recognised and adopted by researchers. This is also evident in the rich results of the numerous $Q$ studies that have been conducted to date and of scholarly literature searches or searches on the $Q$ Methodology website's database for studies. However, some $Q$ studies applied $Q$ methodology as a quantitative tool because of misconceptions about the method's application that regrettably obscure its unique principles (Dziopa and Ahern, 2011). 
The invention of $Q$ methodology has a long and interesting history. William Stephenson (1953), a British physicist and qualified psychologist, not only devised $\mathrm{Q}$ methodology but also introduced its principles to the scientific community. As an assistant to Charles Spearman, a statistician, he used his knowledge of factor analysis to develop the method to uncover subjectivity in any context in a scientific manner (Ramlo, 2016). It can be argued that $Q$ methodology is based on Spearman's traditional method of factor analysis which was adapted as a framework for testing subjectivity (Coogan and Herrington, 2011). After Stephenson introduced Q methodology to the academic community, Steven Brown (1980) from Kent State University became one of the foremost scholars on this methodology in terms of the correct application and analysis of a $Q$ study. Today, Brown (1980) still plays an active role in making the methodology more accessible to scholars who are unfamiliar with the usefulness of studying subjectivity.

$Q$ methodology is a mixed method and uses a meticulous qualitative component (by means of a $Q$ sorting process to rank-order statements) with a quantitative tool ( $Q$ factor analysis) to confirm and analyse similarities in subjective viewpoints ("operant subjectivities") about a topic while isolating the dominant, deeprooted opinions (Du Plessis, 2005; Lee, 2017; Ramlo, 2016). A Q study follows the same rigorous research process of other more conventional methodologies, but is more iterative in that the interpretation of the results could prompt adaptation of, amongst other things, the number of participants required to yield significant factor loadings (Zabala, Sandbrook and Mukherjee, 2018).

Although items for measurement are prepared in the same way as for a survey, Q methodology differs in that individuals and not tests are the variables and it uses an inverted factor technique (Watts and Stenner, 2012). An individual's perceptions or opinions are examined and compared with those of other individuals. Findings are consequently generalised to perspectives, perceptions and opinions, and not to a population (Du Plessis, 2005; Coogan and Herrington, 2011; McKeown and Thomas, 2013; Paige and Morin, 2016).

Q methodology mostly resembles Crossley's narrative analysis (2000) but differs in that participants are invited to engage with a set of prepared items and not their discourse. $Q$ methodology is thus not suitable for the unfolding temporality of narratives (see Popova and Cuffari, 2018), but rather pursues a snapshot of a connected series of attitudes, viewpoints and opinions that are temporally frozen (see Brown, 1980). As a gestalt procedure (Watts and Stenner, 2012), the methodology is distinguished from various forms of discursive or interpretive analyses. Also, when studying highly complex, socially contested and sensitive topics, Q methodology offers a unique starting point (Du Plessis, 2019).

One of the main criticisms levelled against $Q$ methodology, apart from being time-consuming, is that subjective opinions cannot be validated. Although there is some truth in it, $\mathrm{Q}$ methodology nevertheless provides a foundation to structure subjectivity in a way that can be studied and adequately reported on (Brown, 1986).

In this paper, it will be shown through the five steps of a $\mathrm{Q}$ study how social media data could be used to test individuals' attitudes, viewpoints or perceptions based on which statements (generated from a concourse compiled from social media) are meaningful and significant. Participants must be familiar with the topic and have something to say about it. These subjective perspectives are then correlated and transformed into operant factors for in-depth analysis. The factors that are generated do not reflect intuitive or random perceptions, but deep-seated and authentic views (see McKeown and Thomas, 2013).

\section{Proposed steps to follow in a $Q$ study with social media data}

A Q study follows several sequential steps that are interconnected and interdependent, and is thus methodologically well suited to the mixed-methods research approach (Ramlo and Newman, 2011). Although it is similar to traditional research methods, a $Q$ study also requires a feasible research question and a properly executed research design. The following five phases of a $Q$ study were adapted to make provision for using social media data. They are depicted in figure 1 below. 
Feasible Research Question

\begin{tabular}{|c|c|}
$\begin{array}{c}\text { Collecting everything that is } \\
\text { said about the topic in social media } \\
\text { (concourse) }\end{array}$ & $\longrightarrow$ \\
$\begin{array}{c}\text { Data cleaning and organisation with } \\
\text { qualitative data analysis software }\end{array}$ \\
\cline { 2 - 2 }
\end{tabular}

Selecting a representative set of

statements from the organised

concourse for empirical testing

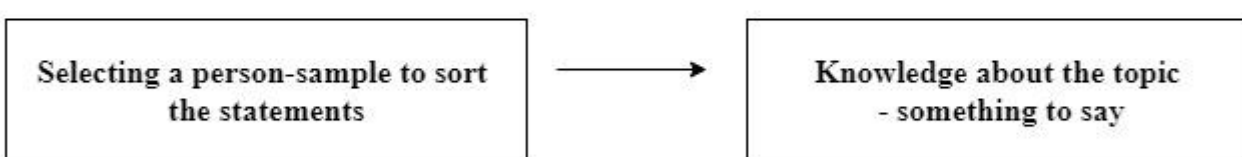

Rank-ordering of the statements

on a grid

Q factor analysis

Figure 1: Proposed steps to follow in a $Q$ study using social media data

\subsection{Collecting everything that is said about the topic in social media (concourse)}

A Q study starts after a researcher has formulated a research question(s) and collected everything that was said about the topic, referred to as the concourse. The concourse of a $Q$ study consists of all the ideas, observations and conversations about a topic, and it could even contain videos, art, music or images collected from primary and/or secondary sources (Brown, 1980).

Using social media data as a concourse for a $Q$ study deserves further exploration. Since social media data are generated by humans in the form of text or image-based communication through computer-mediated webbased services, it can be argued that their subjectivity is expressed through concourses of communication (see McKeown, 1990; Sloan and Quan-Haase, 2017).

A researcher could use a suitable social media data collection tool (some of these tools are web-based whereas others are included in data analysis software) to collect the full extent of opinions and viewpoints on a topic conveyed in a social media network known as a corpus. The corpus becomes the concourse for a social media study and, similarly, includes a collection of written texts, images and videos (Gatto, 2014). Depending on the purpose of the study and the social media network, the concourse may also include hashtags (a \# metadata tag), comments, shares or content which was shared and accessed with a link (Stephen, 2014).

A researcher who uses social media as a data source for a $Q$ study deals with subjective expressions about experiences and views about a topic and not directly with another person. The social media data are raw texts or images by individuals and are separated from the interpretations and potential bias of the researcher (see McKeown, 1990). Nevertheless, an analysis of social media is often considered as superficial because the richness and context of social media users' conversations are not adequately explored. Also, observational data often do not represent all variables in a study and could be manipulated, which might affect a study's results (Poynter, 2018; Carson, 2016). Q methodology could thus become an additional approach to study the current range of attitudes, ideas and beliefs embedded in a concourse consisting of social media data to strengthen results when deemed necessary. 


\subsection{Selecting a representative set of statements from the organised concourse for empirical testing}

Once the full extent of opinions and viewpoints on a topic as conveyed in a social media network (or across social media networks) are captured, the researcher must select a representative set of statements from the concourse (the $Q$ sample) for empirical testing. These statements must ideally be representative of the range of perceptions, opinions and attitudes on the topic.

However, for a Q study using social media data, the researcher must first clean the data of the concourse to ensure accurate results. Proper data cleaning must be followed by initial data analysis with qualitative data analysis software to order and make some sense of the meaning of the vast amount of data. It will be necessary to sift iteratively through the concourse and organise and label different ideas in the same way as would have been done for the social media study. Since data quality is important for reliable results, this essential process will ensure more credible statements for the Q-set (see Chu and Ilyas, 2016).

The study's research question also guides the structure of the set of statements which are referred to as a Qset (Watts and Stenner, 2012). There are two dominant ways of preparing statements for empirical testing since it is not possible to test the entire concourse. The ideas contained in the concourse must be shortened through a data reduction process that can be either structured or unstructured. An unstructured Q-set is more rigorous because statements are grouped into theoretical categories that represent the entire concourse. An unstructured Q-set consists of statements which were randomly selected by the researcher, but which can be easily criticised as being non-representative of the ideas in the concourse (Brown 1980; Ramlo, 2016).

The table below depicts an example of a structured Q sample with 54 statements for a fictitious study on social media communication.

\begin{tabular}{|l|l|l|}
\hline Categories & Levels & Number of levels \\
\hline Reliability & $\begin{array}{l}\text { (a) Narration } \\
\text { (b) Awareness } \\
\text { (c) Community }\end{array}$ & 3 \\
\hline Expectation & $\begin{array}{l}\text { (d) Responsibility } \\
\text { (e) Availability } \\
\text { (f) Relationship }\end{array}$ & 3 \\
\hline Interactions & $\begin{array}{l}\text { (g) Collaboration } \\
\text { (h) Anticipation }\end{array}$ & 2 \\
\hline
\end{tabular}

The fictitious social media communication study applies a factorial design resulting in 18 combinations of the levels (for example, $3 \times 3 \times 2$ ). A set of three statements incorporate the 18 groupings to represent the concourse. A structured $Q$ sample of three statements per level multiplied by 18 totalling 54 statements $(3 \mathrm{X}$ $18=54$ ) is thus constructed (see Du Plessis, 2005). The volume of social media conversations in the concourse requires the researcher to be transparent when capturing and sampling the statements (Stephen, 2014). Fisher's (1960) experimental design to samples can assist in this.

With a structured $Q$ sample, a researcher ensures a focused measurement while, at the same time, placing some boundaries on a topic by using Fisher's (1960) methods of experimental design to the sample (Brown, 1996). While it is recommended for a Q sample to include 40 to 80 statements (Watts and Stenner, 2012), the researcher may also include a post-sort questionnaire to invite comments on participants' sorting choices which can be a rich qualitative input (Muddiman, Bullock, Hampton, Allery, MacDonald, Webb and Pugsley, 2019). Statements are compiled in the same way as for a survey and must be clear, relevant, not doublebarrelled, and written in ordinary language. The statements must also be piloted and if necessary revised before implementation to ensure the internal validity and feasibility of the study (Page and Morin, 2016). 


\subsection{Selecting a person sample to sort the statements}

Since the aim of a $Q$ study is not to generalise findings to a larger population but to obtain a range of perspectives on a topic, a $Q$ study does not require as many participants (known as a person sample) as for a survey in order to yield statistical significance. Representativeness is rather sought for the Q-set of statements. The number of participants typically varies between 40 and 60 individuals to identify significant factors.

However, Q studies with fewer participants yielding significant factors are available in the body of literature. A person sample for a $Q$ study is often purposively selected based on people's interest in or knowledge of a topic (Brown, 1980; Du Plessis, 2005; Watts and Stenner, 2012).

A researcher involved in a social media study must have focused selection criteria to invite and select participants to benefit the study's research question(s) by ensuring a variety of perspectives (see Watts and Stenner, 2012). As Brown (2017) points out, different viewpoints have already existed before the $Q$ study, and the researcher must adopt a strategy that ensures that these viewpoints load as factors and which starts with the structured compilation of the Q-set and continues with the person sample. Because most participants will not be familiar with $Q$ methodology, ensuring a vested interest in the topic with a suitable sample frame could enhance the desired number of participants (see Lee, 2017).

The more diverse participants' perspectives are, the more information-rich and significant the results will be (Watts and Stenner, 2012). Since web-based software is available to conduct a $Q$ study, the person sample can nowadays include participants across the globe. However, as pointed out by Du Plessis (2019), to conduct an online $Q$ study, researchers must ensure that $Q$ software is frequently updated and compatible with any computer's operating system.

\subsection{Rank-ordering of the statements on a grid}

Participants for the person sample are involved in a $Q$ sorting process to rank-order numbered statements (the Q-set) from their viewpoints. Before participants start with the sorting process, ethical considerations are explained and informed consent is given to participate in the study. The sorting of the statements represents individual responses to the statements following a "condition of instruction" which depends on the type of study and the research question. Participants may be required to sort the statements according to a preset grid with a space for each statement on the grid (forced choice). Another option is to allow participants to sort the statements with a "free-sort" instruction anywhere on the grid (McKeown and Thomas, 2013; Lee, 2017).

However, "forcing" participants to sort statements on a preset grid makes the statements more stable and discriminating (Lee, 2017). Irrespective of whether or not a participant agrees with all the statements, ranking is feasible (see Barry and Proops, 1999). The grid has a Likert scale format which typically includes a five to nine-point relative but not absolute scale to indicate participants' agreement or disagreement on a statement (Brown, 1980).

The numbered statements are first sorted into three piles regarding agreement, disagreement and being neutral before being placed on the grid with an opportunity for the participant to reconsider the initial sorting at a later stage (Brown, 1980). However, with a free-sort condition of instruction, participants are unhindered and may place the statements anywhere on a $Q$ sort grid. The statements can also be sorted into more than three piles before placement on the grid commences. The free sorting process could increase bias, even unintentionally, and is not widely adopted (Du Plessis, 2005).

While participants in a $Q$ study may still sort the statements offline if preferred, thanks to advanced new technologies, Q methodology allows for an online $Q$ sorting process with user-friendly interfaces making the method faster and simpler (Pruslow and Red Owl, 2012). But more could be done to accommodate a social media concourse as put forward by Stephen (2014).

The figure below is an example of a preset grid with space for 40 statements with a nine-point scale. 


\begin{tabular}{|c|c|c|c|c|c|c|c|c|}
\hline-4 & -3 & -2 & -1 & 0 & +1 & +2 & +3 & +4 \\
\hline 8 & 3 & 15 & 14 & 1 & 4 & 23 & 6 & 9 \\
\hline \multirow[t]{7}{*}{34} & 22 & 40 & 35 & 32 & 37 & 17 & 2 & 33 \\
\hline & \multirow[t]{6}{*}{21} & 20 & 28 & 13 & 10 & 26 & 19 & \\
\hline & & 7 & 31 & 5 & 12 & 27 & & \\
\hline & & \multirow[t]{4}{*}{36} & 11 & 24 & 29 & 38 & & \\
\hline & & & 18 & 30 & 39 & & & \\
\hline & & & & 16 & & & & \\
\hline & & & & 25 & & & & \\
\hline
\end{tabular}

Figure 1: A preset $Q$ sort grid representing the numbers of the statements as they were placed on the grid.

\section{5 $Q$ factor analysis}

The $Q$ factor analysis is the last step in a $Q$ study and is often referred to as inverse factor analysis since the data matrix is turned on its side (Rozalia, 2008). During this step, correlations between participants' perspectives and not variables are factored and clustered together. The Horst centroid method is used to compute and factor analyse the intercorrelations between the different $Q$ sorts. The number of factors that is extracted is based on the limiting level of "residual correlations" (Horst, 1962). Resulting unrotated factors can be rotated with the Varimax or Judgemental rotation. Varimax rotation constitutes an analytic procedure whereas Judgemental rotation is done manually and is theoretically driven (Du Plessis, 2005). Pure factor loadings ( $Q$ sorts that load only on one factor) should be a consideration for analysis since these factors provide a focused perspective of a topic (Brown, 1996). Further considerations for analysis are a factor's eigenvalues, distinguishing statements as well as the number of participants that load on a factor.

Distinguishing statements are those statements that reflect the major differences between the factors and could assist the researcher with interpretation (Coogan and Herrington, 2011).

Table 1: An example of the factor variance and eigenvalues of a $Q$ study

\begin{tabular}{|l|c|c|c|c|}
\hline Factor & 1 & 2 & 3 & Total \\
\hline Eigenvalues & 5.8 & 2.3 & 1.6 & 9.7 \\
\hline $\begin{array}{l}\text { \% explained } \\
\text { variance }\end{array}$ & 15 & 12 & 9 & 36 \\
\hline
\end{tabular}

\section{Discussion}

This article advances the combination of $\mathrm{Q}$ methodology and social media research that uses observational social media data containing multifaceted, sensitive or controversial topics. It is argued that while technologies offer numerous new research opportunities and advantages, researchers also face challenges, especially when it comes to the analysis of a vast amount of social media data that might consist of fake profiles, manipulated information and spam (Tufekci, 2014). Without the use of advanced software to adequately clean the data from bogus information, results might differ completely from that of a social media study relying on adequately cleaned data (Zheng et al., 2015). The rapid availability of new knowledge means that erroneous results could have a profound effect on the academic discourse and even change reality (Casadevall and Fang, 2012). For this reason, $Q$ methodology could provide a robust technique to enhance the results of a social media study before novel ideas that could change theory and practice are published. However, the researcher needs to adequately and transparently capture and sample the range of ideas in the concourse and recruit participants that have a useful opinion about the topic (Stephen, 2014).

Without a doubt, the context of a social media study plays a role when it comes to the strengths and limitations of combining the study with $Q$ methodology. $Q$ methodology could also be useful when a social media study requires evidence of rigor and transparency. It could also provide deep insight into a social media topic which traditional research methods cannot provide (see Bashatah, 2016). The method allows for great 
insight into how individuals feel about a topic that plays out in social media but does not necessarily represent the views of the larger online and offline population. In addition, an authentic voice can be added to support new ideas that could spark further debate (see Hughes, 2012; Poynter, 2018).

Using $\mathrm{Q}$ methodology to test and validate perspectives and attitudes evident in social media data can be a rewarding experience for both the researcher and the participant. Participants will provide honest and authentic viewpoints about a topic in which they are interested, and the researcher will obtain rich data without fear of bias in a study (see Bashatah, 2016). However, the researcher will need to carefully consider who the participants in a $Q$ study with social media data will be, and decide on the most efficient way to recruit them (Stephen, 2014).

Since researchers set great store on access to new technologies and the ease with which they can study and report on previously unattainable topics (Shalin, 2015), it is acknowledged that combining a social media study with $\mathrm{Q}$ methodology could make the study more time-consuming. This should, however, not deter social media researchers to enrich their studies with a unique alternative method. Supplementing social media research with $Q$ methodology could be a functional add-on and an empowering experience since it highlights different and similar views about a topic, integrate diverse offline and online views while affording opportunities for further research and debate. In addition, rich primary data that are both objective and balanced are collected (see Ramlo, 2005; 2016).

\section{Conclusion}

This paper draws on the conception of $Q$ methodology as argued by experienced scholars. The idea that more social media researchers should consider enhancing their studies with $Q$ methodology when a topic under investigation yields a large amount of potentially tainted social media data, was put forward. This procedure should be especially useful for topics that attract attention in social media, but which do not necessarily reflect views in the real offline world. Consequently, $\mathrm{Q}$ methodology could be a valuable complementary research tool to not only uncover and validate but to integrate offline and online perspectives for more profound insight. The discussion was illustrated by referring to the five phases of a $Q$ study which were tailored to accommodate a large social media dataset. Numerous $Q$ studies about social media have been conducted, but none of them uses a large social media dataset as its main concourse, which could be a valuable addition to social media research.

The paper expands our knowledge of how social media studies could be augmented with Q methodology and also highlights potential difficulties. Because the scope of social media technologies is everchanging and opportunities for research are on the increase, $Q$ methodology could enhance its legitimacy as a useful methodology by adapting its techniques to fully embrace the social media environment. Even though $Q$ methodology was modified over the years to have the capacity for an online environment, more can be done to accommodate large social media datasets as the concourse. Future studies could, therefore, modify the proposed views in this paper by incorporating more practical ideas or proposing ways in which Q methodology technologies could be adapted to benefit social media research in the 4IR era.

\section{References}

Angelopulo G., 2009. Q methodology and the measurement of subjectivity in corporate brand perception. South African Journal of Business Management, 40(3), pp. 21-33.

Barry, J., and Proops, J., 1999. Seeking sustainability discourses with Q methodology. Ecological Economics, 28(33), pp. 337-345.

Bashatah, L.S., 2016. Q-methodology: What and how? Journal of Research \& Method in Education, 6(5), pp. 37-43.

Beninger, K., Fry, A., Jago, N., Lepps, H., Nass, L., and Silvester, H., 2014. Research using Social Media Users' Views. NatCen Social Research, pp. 1-40.

Brown, S.R., 1980. Political subjectivity: Applications of Q methodology in Political Science. New Haven, CT: Yale University Press.

Brown, S.R., 1986. Q technique and method: Principles and procedures. In W.D. Berry and M.S. Lewis-Beck, eds. New tools for social scientists: Advances and applications in research methods. Beverly Hills, CA, SAGE Publications Ltd. pp. 5776.

Brown, S.R., 1996. Q methodology and qualitative research. Qualitative Health Research, 6(4), pp. 61-567.

Brown, S.R., 2017. Inquiry about determining the number of participants in the P-set [online] 3 October 2017. Available at: q-method@listserv.kent.edu [Accessed 31 July 2019].

Burgess, J., Marwick, A., and Poell, T. (Eds)., 2017. The SAGE Handbook of Social Media. UK: SAGE Publications Ltd. 
Burke, Lydia, C-A., 2015. Exploiting the qualitative potential of Q methodology in a post-colonial critical discourse analysis. International Journal of Qualitative Methods, 14(1), pp. 65-79.

Carson, B., 2016. Social Media as a Research Methodology. Market Research, [blog] 8 December. Available at: https://blog.marketresearch.com/social-media-as-a-research-methodology [Accessed 31 July 2019].

Casadevall, A., and Fang, F.C., 2012. Reforming Science: Methodological and cultural reforms. Infection and Immunity, 80(3), pp. 891-896.

Chu, X., and Ilyas, I.F., 2016. Qualitative data cleaning. In: Proceedings of the VLDB Endowment, 9(13), pp. 1605-1608. Available at: http://www.vldb.org/pvldb/ [Accessed 31 July 2019].

Coogen, J., and Herrington, N., 2011. Q methodology: an overview. Research in Secondary Teacher Education, 1(2), pp. 2428.

Cross, R.M., 2004. Exploring attitudes: The case for Q methodology. Health Education Research, 20(2), pp. 206-213.

Crossley, M.L., 2000. Introducing narrative psychology: Self, trauma and the construction of meaning. Open University Press: Buckinghamshire.

Cureton, E.E., and D'Agostino, R.B., 1993. Factor analysis: an applied approach. United States: Lawrence Erlbaum Associates.

Dimitrios S., and Airey, D., 2011. Q-methodology and tourism research. Current Issues in Tourism, 14(4), pp. 311-322.

Du Plessis, C., 2005. A theoretical framework of corporate online communication: A marketing public relations (MPR) perspective. DPhil. University of South Africa. Available at: http://hdl.handle.net/10500/2271 [Accessed 31.07.2019].

Du Plessis, C., 2019. Using Q methodology to test Perspectives and Attitudes: Experiences from a Study about Content Marketing. In: Proceedings of 18th European Conference on Research Methodology for Business and Management Studies. Johannesburg, South Africa, 20-21 June 2019. UK: ACPI.

Dziopa, F., and Ahern, K., 2011. A systematic literature review of the applications of Q-technique and its methodology. Methodology, 7(2), pp. 39-55.

Ellingsen, I.T., St $\varnothing$ rksen, I and Stephens, P., 2010. Q methodology in social work research. International Journal of Social Research Methodology, 13(5), pp. 395-409.

Emmel, N., 2013. Sampling and choosing cases in qualitative research: A realist approach. London: SAGE Publications Ltd.

Gatto, M., 2014. Web as corpus. Theory and Practice. London: Bloomsbury.

Grover, V.K., 2015. Developing indicators of quality school education as perceived by teachers using Q-methodology approach. Zenith International Journal of Multidisciplinary Research, 5(8), pp. 54-65.

Hensel, D., 2016. Q-Methodology: An Alternative Design for Undergraduate Nursing Honors Research. Journal of Nursing Education, 55(11), pp. 659-662.

Hughes, M., 2012. Researching behaviour: A Q methodological exploration of the position of the young person as researcher. DEd. University of Sheffield. Available at: http://etheses.whiterose.ac.uk/7737/1/FINAL\%20thesis\%20April\%2029\%202012.pdf [Accessed 20 July 2019].

Inuwa-Dutse, I., Liptrott, M., and Korkontzelos, I., 2018. Detection of spam-posting accounts on Twitter. Neurocomputing, 315, pp. 496-511.

Kampen, J.K., and Tamás, P., 2014. Overly ambitious: contributions and current status of Q methodology. Quality Quantity, 48(6), pp. 3109-3126.

Killam, L., Timmermans, K.E., and Raymond, J.M., 2013. The barriers to and benefits of conducting Q-sorts in the classroom Nurse Researcher, 21(2), pp. 24-29.

Lee, B.S., 2017. The fundamentals of Q methodology. Journal of Research Methodology, 2(2), pp. 57-95.

Le Roux, C., and Du Plessis, C., 2014. An exploratory Q study of corporate brand identity elements governing corporate brand image formation. Southern African Business Review, 18(3), pp. 119-141.

McKeown, B.F., and Thomas, D.B., 2013. Q methodology (Quantitative Applications in the Social Sciences Series). Second Edition. Newbury Park, CA: SAGE Publications Ltd.

McKeown, B.F., 1990. Q methodology, communication, and the behavioral text. The Electronic Journal of Communication, $1(1)$.

Muddiman, E., Bullock, A..D., Hampton, J.M., Allery, L., MacDonald, J., Webb, K.L. and Pugsley, L. 2019. Disciplinary boundaries and integrating care: using Q-methodology to understand trainee views on being a good doctor. $B M C$ Medical Education, 19(59).

Orchard, L.F., Fullwood, C., Galbraith, N., and Morris, N., 2015. Investigating the Facebook experience through Q Methodology: Collective investment and a 'Borg' mentality. New Media and Society, 17(9).

Paige, J.B., and Morin K.H., 2016. Q-Sample Construction: A Critical Step for a Q-Methodological Study. Western Journal of Nursing Research, 38(1), pp. 96-110.

Parker, J., and Alford, C., 2010. How to use Q-methodology in dream research: assumptions, procedures and benefits. Dreaming, 20(3), pp. 169-183.

Popova, Y., and Cuffari, E., 2018. Temporality of sense-making in narrative Interactions. Cognitive Semiotics, 11(1), Available at: https://doi.org/10.1515/cogsem-2018-0007.

Poynter, R., 2018. Researchers Should Be Aware of the Problems With Observational Data. Greenbook blog, [blog] $31 \mathrm{May}$ 2018. Available at: ,https://greenbookblog.org/2018/05/31/researchers-should-be-aware-of-the-problems-withobservational-data> [Accessed 31 July 2019].

Pruslow, J.T., and Red Owl, R.H., 2012. Demonstrating the application of Q methodology for fieldwork reporting in experiential education. Journal of Experiential Education, 35(2), pp. 375-392. 
Ramlo, S. 2005. An application of Q methodology: Determining college faculty perspectives and consensus regarding the creation of a school of technology. Journal of Research in Education, 15(1), pp. 52-69.

Ramlo, S.E., and Newman, I., 2011. Q Methodology and Its Position in the Mixed-Methods Continuum. Operant Subjectivity, 34(3), pp. 172-191.

Ramlo, S., 2016. Mixed Method Lessons Learned From 80 Years of Q Methodology. Journal of Mixed Methods Research, 10(1), pp. 28-45.

Richardson, L. A., 2016. Weights: An exploration of university exercise science students' views of obesity. Ph.D. University of Akron. Available at: https://etd.ohiolink.edu/pg_10?::NO:10:P10_ETD_SUBID:113826 [Accessed 14.08.2019].

Rozalia, G.M., 2008. Q factor analysis (Q-methodology) as data analysis technique, Annals of the University of Oradea. Economic Science Series, 17(4), pp. 871-876.

Sappleton, N. (ed)., 2013. Advancing Research Methods with New Technologies. United States of America: Information Science Reference.

Schmolck, P., 2008. Common and specific approaches in the analysis of Q-sort data with PQ Method. Keynote speech presented at the 24th Annual Q Conference. Hamilton, Ontario. Available: http://schmolck.org/qmethod/Schmolck_Hamilton-2008_Talk.ppt [Accessed 14 August 2019].

Schwab. K., 2016. The Fourth Industrial Revolution. New South Wales, Australia: Currency.

Shalin, H-J., 2015. Enhancing Qualitative and Mixed Methods Research with Technology. United States of America: Information Science Reference.

Sloan, L., and Quan-Haase, A. (eds)., 2019. The SAGE Handbook of Social Media Research Methods. Los Angeles: SAGE Publications Ltd.

Stephen, J., 2014. Interpreting hashtag politics: Policy ideas in an era of social media. London: Palgrave Macmillan. Stephenson, W., 1953. The study of behavior: Q-technique and its methodology. Chicago: University of Chicago Press. Stergiou, D., and Airey, D., 2011. Q-methodology and tourism research. Current Issues in Tourism, 14(4), pp.311-322.

Sung-Hee, L., Euna, P., and Myung-Ae, K., 2014. The Typology of Gender Role Identity in Middle-aged Women using Q Methodology. Journal of Digital Convergence, 12(6), pp. 449-456.

Thomson, T.J., and Greenwood, K. 2017. I "Like" That: Exploring the Characteristics That Promote Social Media Engagement With News Photographs. Visual Communication Quarterly, 24(:4), pp. 203-218.

Tufekci, Z., 2014. Big Questions for Social Media Big Data: Representativeness, Validity and Other Methodological Pitfalls. In: ICWSM '14: Proceedings of the 8th International AAAI Conference on Weblogs and Social Media, Michigan, USA. 1-4 June 2014. USA: AAAI Press.

Watts, S., and Stenner, P., 2012. Doing Q Methodological Research: Theory, Method \& Interpretation. London: SAGE Publication Ltd.

Zabala, A., Sandbrook, C., and Mukherjee, N., 2018. When and how to use Q methodology to understand perspectives in conservation research. Conversation Biology 32(5), pp. 1185-1194.

Zheng, X., Zeng, Z., Chen, Z., and Rong, C., 2015. Detecting spammers on social networks. Neurocomputing, 159, pp. 27-34. 